\title{
Postoperatively Determined High-Risk Histopathologic Features in Papillary Thyroid Carcinoma Initially Eligible for Thyroid Lobectomy: A Game Changer
}

\section{Sohail Bakkar ( $\nabla$ sohail.bakkar@gmail.com )}

The Hashemite University Faculty of Medicine https://orcid.org/0000-0001-8479-5901

\section{Khaled Al-Omar}

The Hashemite University Faculty of Medicine

\section{Gianluca Donatini}

University of Poitiers: Universite de Poitiers

\section{Qusai Aljarrah}

Jordan University of Science and Technology

Theodosios S Papavramidis

Aristotle University of Thessaloniki: Aristoteleio Panepistemio Thessalonikes

\section{Gabriele Materazzi}

Pisa University Hospital: Azienda Ospedaliero Universitaria Pisana

\section{Paolo Miccoli}

Pisana University Hospital: Azienda Ospedaliero Universitaria Pisana

\section{Research Article}

Keywords: Low-risk, Papillary thyroid carcinoma, Extent of surgery, Total thyroidectomy, Thyroid lobectomy.

Posted Date: April 13th, 2021

DOI: https://doi.org/10.21203/rs.3.rs-390654/v1

License: (c) (i) This work is licensed under a Creative Commons Attribution 4.0 International License. Read Full License 


\section{Abstract \\ Purpose.}

Recent clinical practice guidelines consider thyroid lobectomy a viable alternative for low-risk papillary thyroid carcinoma PTC measuring 1-4 cm in size. We aimed to assess the likelihood of finding postoperatively determined high-risk histopathologic features that would lead to the recommendation of completion thyroidectomy.

\section{Methods.}

A retrospective review of patients who underwent total thyroidectomy for PTC measuring $1-4 \mathrm{~cm}$ in size between Jan 2012-Jan 2018 was conducted. Patients with pre-operative high-risk characteristics were excluded; history of radiation exposure, positive family history, clinically suspicious cervical lymphadenopathy, and gross extrathyroidal extension (ETE). A hypothetical group of 245 patients remained eligible for lobectomy. The pathology specimens from the cancer-containing lobes were evaluated for high-risk features: aggressive histology, capsular and/or vascular invasion, microscopic ETE, and multifocality. A subgroup analysis was performed with $2 \mathrm{~cm}$ being the cut-off size.

\section{Results.}

The average age was 39 years with $73 \%$ being females. Mean cancer size was $16 \mathrm{~mm}$. Evaluation of the cancer-containing lobe for high-risk features revealed: aggressive histology (33\%), ETE (12\%), capsular invasion (33\%), vascular invasion (17\%), and ipsilateral multifocality (30\%). The cumulative risk of having $\geq 1$ high-risk feature mandating completion thyroidectomy was $59 \%$. The risk was considerably higher for lesions $\leq 2 \mathrm{~cm}$ compared to larger lesions ( $64 \%$ vs.48\%; $p=0.049 ; \mathrm{RR}=1.3$ ).

\section{Conclusion.}

A considerable proportion of patients initially eligible for lobectomy have high-risk features that only become evident at pathology. Therefore, a comprehensive approach is advocated to determine the extent of surgery for PTC incorporating patient preferences regarding risks and benefits.

\section{Introduction}

Papillary thyroid carcinoma (PTC) is the most common primary malignancy of the thyroid gland. Its incidence has been rising rapidly and steadily, and is postulated to soon become the third most common cancer among women worldwide [1, 2]. Therefore, its optimal management has been gaining increasing popularity among healthcare providers, particularly the extent of the initial surgery required. To date, this remains an area of ongoing controversy. 
The most recent clinical practice guidelines; the American Thyroid Association (ATA), National Comprehensive Cancer Network (NCCN), and the British Thyroid Association (BTA), consider thyroid lobectomy as sufficient surgery for differentiated thyroid cancer up to $4 \mathrm{~cm}$ in size in low-risk patients with low-risk tumors. While total thyroidectomy and/or completion thyroidectomy remain the standard of care in high-risk patients with high-risk tumors [3]. This is otherwise related to the indolent nature of these tumors and their favorable prognosis. According to ATA, high-risk histologic features include aggressive histologic variants, multifocality, microscopic extrathyroidal extension, and metastatic lymphadenopathy [4]. The purpose of this study was to assess the likelihood of finding postoperatively determined high-risk histopathologic features that would lead to the recommendation of completion thyroidectomy and its implication on current clinical practice recommendations

\section{Materials And Methods}

Between Jan. 2012 and Jan. 2018, 324 patients underwent total thyroidectomy for PTC measuring 1-4 $\mathrm{cm}$ in size. A retrospective analysis of prospectively maintained data was performed excluding patients with pre-operative high-risk characteristics in concordance with the most recent clinical practice guidelines, namely: the ATA guidelines for the management of adults with differentiated thyroid cancer, the NCCN, and BTA guidelines [3]. Pre-operative high-risk characteristics included: history of radiation exposure, a positive family history of thyroid cancer or thyroid cancer syndrome, clinically suspicious cervical nodal metastasis, and sonographically detected gross extrathyroidal extension. Patients above the age of 55 years were also excluded in concordance with the 8th edition of the American Joint Committee on Cancer (AJCC) TNM staging system for differentiated thyroid cancer and anaplastic thyroid cancer that uses the age of 55 years as the new cut-off age for risk stratification [5]. A hypothetical group of 245 patients remained eligible for thyroid lobectomy. The pathology specimens from the cancer-containing lobes were then evaluated for high-risk histologic features, including: aggressive histology, capsular and/or vascular invasion, microscopic extrathyroidal extension, and ipsilateral multifocality as per ATA guidelines [4]. Nodal metastasis was not evaluated as our institute's policy in dealing with cervical lymph nodes is a therapeutic selective compartment-oriented functional neck dissection based on clinically suspicious lymphadenopathy (sonographically or as an intraoperative finding), as the role of prophylactic neck dissection in the management of PTC remains controversial regarding its risks and benefits [6]. Furthermore, cases with suspicious lymphadenopathy would not be considered eligible for thyroid lobectomy and were excluded. Innovative advanced energy-based devices were used routinely for their potential benefits in thyroid surgery [7]. PTC histologic variants labeled as aggressive included tall-cell, columnar cell, solid, and diffuse sclerosing variants. Contralateral multifocality was also evaluated as a potentially invisible feature that would preclude thyroid lobectomy. The study cohort was then segregated into two groups based on tumor size with $2 \mathrm{~cm}$ being the cut-off size, and a subgroup analysis for the primary outcome of interest was performed.

Data collection and analysis were performed according to the institutional guidelines and the ethical standards of the Helsinki Declaration and its amendments. The requirement for an informed consent was waived because of the retrospective nature of the study. 
Categorical data were described by absolute and relative frequency, continuous data by mean and standard deviation. To compare quantitative variables with qualitative variables $t$-test for independent samples (two-tailed) was used, whereas to compare qualitative variables Fisher's exact test and z-test for two proportions were applied. Statistical significance was defined as a $p$ value $<0.05$. All analyses were performed by SPSS 2017 v. 25 software.

\section{Results}

The study cohort consisted of 178 females (73\%), and 67 males (27\%) with a female to male ratio of 3:1. The mean age was 39 years (range: $14-55$ years). The mean cancer size was $16 \mathrm{~mm}$ (range: 10-40 $\mathrm{mm})$. Evaluation of the cancer-containing lobe revealed: aggressive histology in 33\% (81/245) of cases, microscopic extrathyroidal extension in $12 \%(29 / 245)$ of cases, capsular invasion in $33 \%(81 / 245)$ of cases, vascular invasion in 17\% (42/245) of cases, and ipsilateral multifocality in $30 \%$ (73/245) of cases. Contralateral multifocality occurred in $29 \%(72 / 245)$ of cases (Table 1$)$. Aggressive histologic variants included: any PTC with $\geq 10 \%$ of tall-cells $(26.5 \%)$, solid variant $(5 \%)$, columnar-cell variant $(1 \%)$, and diffuse sclerosing variant of PTC $(0.5 \%)$. Low-risk variants included: the classical variant $(62 \%)$, and the follicular variant of PTC (5\%). The follicular variant of PTC was considerably more frequent among lesions measuring $>2 \mathrm{~cm}$ in size $(11.5 \%$ vs. $3.5 \% ; p=0.03)$ (Table 2$)$.

Table 1

The proportion of postoperatively determined high-risk histopathologic features in PTC lesions $1-4 \mathrm{~cm}$ in size, and in the subgroups of lesions with $2 \mathrm{~cm}$ being the cut-off size.

\begin{tabular}{|lllll|}
\hline Histopathologic feature & $\mathbf{1 - 4} \mathbf{~ c m ~}(\mathbf{n}=\mathbf{2 4 5})$ & $\mathbf{5} \mathbf{~ c m ~}(\mathbf{n}=\mathbf{2 0 1})$ & $\mathbf{> 2} \mathbf{~ c m}-\mathbf{4} \mathbf{~ c m}(\mathbf{n}=\mathbf{4 4})$ & $\boldsymbol{p}$-value \\
\hline Aggressive histology & $33 \%(81 / 245)$ & $35 \%(70 / 201)$ & $52 \%(23 / 44)$ & 0.035 \\
\hline Extrathyroidal extension & $12 \%(29 / 245)$ & $12 \%(25 / 201)$ & $9 \%(4 / 44)$ & 0.57 \\
\hline Capsular invasion & $33 \%(81 / 245)$ & $34 \%(68 / 201)$ & $29.5 \%(13 / 44)$ & 0.56 \\
\hline Vascular invasion & $17 \%(42 / 245)$ & $17 \%(35 / 201)$ & $16 \%(7 / 44)$ & 0.87 \\
\hline Ipsilateral multifocality & $30 \%(73 / 245)$ & $31 \%(62 / 201)$ & $25 \%(11 / 44)$ & 0.50 \\
\hline Contralateral multifocality & $29 \%(72 / 245)$ & $30 \%(60 / 201)$ & $27 \%(12 / 44)$ & 0.79 \\
\hline Cumulative risk & $59 \%$ & $64 \%$ & $48 \%$ & 0.049 \\
\hline The $p$-value column represents a comparison between the two subgroups of lesions. & \\
\hline
\end{tabular}


Table 2

The proportions of the various histologic variants of papillary thyroid carcinoma (PTC), and in the subgroups of lesions with $2 \mathrm{~cm}$ being the cut-off size.

\begin{tabular}{|c|c|c|c|c|}
\hline Histologic variant & $\begin{array}{l}1-4 \mathrm{~cm}(n= \\
245)\end{array}$ & $\begin{array}{l}\leq 2 \mathrm{~cm}(\mathrm{n}= \\
201)\end{array}$ & $\begin{array}{l}>2 \mathrm{~cm}-4 \mathrm{~cm}(\mathrm{n}= \\
44)\end{array}$ & $\begin{array}{l}p- \\
\text { value }\end{array}$ \\
\hline Classical PTC & $62 \%(152 / 245)$ & $\begin{array}{l}62 \% \\
(124 / 201)\end{array}$ & $64 \%(28 / 44)$ & 0.8 \\
\hline FVPTC & $5 \%(12 / 245)$ & $3.5 \%(7 / 201)$ & $11.5 \%(5 / 44)$ & 0.03 \\
\hline Tall cell variant & $\begin{array}{l}26.5 \% \\
(65 / 245)\end{array}$ & $\begin{array}{l}28.5 \% \\
(57 / 201)\end{array}$ & $18.5 \%(8 / 44)$ & 0.17 \\
\hline Solid variant & $5 \%(12 / 245)$ & $5 \%(11 / 201)$ & $2 \%(1 / 44)$ & 0.38 \\
\hline $\begin{array}{l}\text { Columnar cell } \\
\text { variant }\end{array}$ & $1 \%(3 / 245)$ & $1 \%(2 / 201)$ & $2 \%(1 / 44)$ & 0.57 \\
\hline DSV-PTC & $0.5 \%(1 / 245)$ & $0 \%(0 / 201)$ & $2 \%(1 / 44)$ & 0.045 \\
\hline
\end{tabular}

Combining postoperatively determined high-risk histopathologic features, the cumulative risk of having $\geq$ 1 high-risk feature for PTC lesions 1-4 cm in size was 59\% (145/245). Out of 245 patients, 201 (82\%) had PTC lesions $\leq 2 \mathrm{~cm}$ in size, and 44 patients had lesions $>2 \mathrm{~cm}$ and up to $4 \mathrm{~cm}$ in size. Subgroup analysis yielded a cumulative risk of having $\geq 1$ high-risk feature for lesions $\leq 2 \mathrm{~cm}$ of $64 \%(128 / 201)$. While the risk was $48 \%(21 / 44)$ for lesions $>2 \mathrm{~cm}$ and up to $4 \mathrm{~cm}$ in size. The cumulative risk of having $\geq$ 1 high-risk feature that would lead to the recommendation of completion thyroidectomy was considerably lower for lesions larger than $2 \mathrm{~cm}$ in size ( $p$-value $=0.049,95 \% \mathrm{Cl}$ : $0.13-31.35 \%, \mathrm{RR}=1.3)$ (Table 1$)$. The likelihood of having $\geq 1$ high-risk feature was not influenced by gender; neither in the entire study cohort nor in the two designated subgroups.

\section{Discussion}

The fundamental principles of surgical oncology prioritize adequate loco-regional control with a cautious attitude towards radical intervention, because of the potential for increased morbidity [8]. These principles are in line with the time-honored motto of Medicine "primum non nocere" or "first, do no harm" [9].

When managing well differentiated thyroid cancer, surgical adequacy is the most important prognosticator. Whereas other treatment modalities (radioactive iodine ablation and hormone suppressive therapy) are only considered adjunct therapies. Nevertheless, the indolent nature of these tumors has led to a paradigm shift towards more conservative and less morbid surgery. Yet, total thyroidectomy has remained the standard of care for high-risk patients with high-risk tumors [6]. In this study, we sought to assess the likelihood of finding postoperatively determined high-risk histopathologic features that would lead to the recommendation of completion thyroidectomy and its implication on current clinical practice recommendations. 
The study by Bilimoria et al, a population-based study, analyzed 61,775 patients from the National Cancer Data Base (NCDB) and advocated total thyroidectomy for differentiated thyroid cancer $>1 \mathrm{~cm}$ in size [9]. It served as the basis for the management recommendations for differentiated thyroid cancer of the 2009 version of the ATA guidelines [10]. The paradigm shift in the extent of thyroid surgery for PTC was largely based on the study by Adam et al. who analyzed the same data after adjusting for several patient, clinical and tumor-related prognostic factors that weathered the limitations of the previous study [12].

Furthermore, a recent analysis of the impact of the extent of thyroid surgery in 43,032 patients under the age of 45 years collected from the two largest oncology data bases: the NCDB and the Surveillance Epidemiology and End Results (SEER) database, underscored the prognostic significance of low-risk disease and confirmed the 7th edition of the AJCC staging system for differentiated thyroid cancer in lowrisk patients [13]. The 7th of the AJCC staging system classifies these patients as having only stage I or II disease, based solely on the absence or presence of distant metastasis, respectively, regardless of the size of their tumors or extent of loco-regional disease [14]. This also applies to the latest edition of this staging system [5].

A recent large retrospective study evaluated the effect of the extent of thyroid surgery on the oncologic outcome in patients with differentiated thyroid cancer $1-4 \mathrm{~cm}$ in size in terms of disease-specific survival and disease-free survival [15]. It concluded that the extent of thyroid surgery was an independent risk factor for disease-free survival and that total thyroidectomy had a favorable impact in reducing recurrent disease [15]. These findings were directly related to post-operatively determined high-risk cancerous histologic features. Our findings are in line with these results. Although our study does not provide a comparative analysis regarding disease-free survival, it clearly demonstrates a high proportion of postoperatively determined high-risk histopathologic features that correlate with disease-free survival. Patients initially eligible for thyroid lobectomy had cumulative risk of $59 \%$ of having $\geq 1$ high-risk feature. Our study further expanded to include all potential high-risk features stated in clinical practice guidelines: aggressive histologic variants of PTC (33\%), microscopic extrathyroidal extension (12\%), capsular invasion (33\%), vascular invasion (17\%), and ipsilateral multifocality (30\%). We further highlighted the impact of a potential invisible enemy (contralateral disease) which occurred in $29 \%$ of the study cohort. By conducting a two-group designation based on size with $2 \mathrm{~cm}$ being the cut-off, our results suggest that a small tumor size should not give surgeons a false sense of security selecting conservative surgery. As the cumulative risk of having $\geq 1$ high-risk feature that would lead to the recommendation of completion thyroidectomy was considerably higher for lesions smaller than $2 \mathrm{~cm}$ in size ( $p$-value $=0.049$, $95 \% \mathrm{Cl}: 0.13-31.35 \%, \mathrm{RR}=1.3)$. Another aspect to consider when selecting patients for a conservative initial surgery as per changed international clinical practice guidelines, is the potential additional risk and inconvenience brought about by completion thyroidectomy on the overall patient experience [16].

Limitation of this study include its retrospective nature, and the relatively small sample size of lesions $>2$ and up to $4 \mathrm{~cm}$ in size which potentially underpowers group comparisons. Furthermore, an ideal assessment would require a prospective randomized study with follow-up data on disease-free survival which is the main outcome of interest in surgery for PTC. 


\section{Conclusion}

With the lack of prospective randomized controlled trials on the extent of thyroid surgery for PTC 1-4 cm in size in literature, the optimal surgical strategy for these tumors is yet to be established. However, as a considerable proportion of patients initially eligible for lobectomy, as per current clinical practice guidelines, have high-risk features that only become evident at pathology, a comprehensive approach is advocated to determine the extent of surgery incorporating patient preferences regarding risks and benefits.

\section{Declarations}

Author contributions corresponding author SB: study concept and design, data interpretation, article writing, final approval, accountability for all aspects of the work. KA, GD, QA and TP: data collection and interpretation, final approval, accountability for all aspects of the work. GM, PM: critical revision, final approval, accountability for all aspects of the work.

\section{Data availability statement}

The data supporting the findings of this study are available from the corresponding author upon request.

\section{Compliance with ethical standards}

\section{Conflict of interest}

The authors declare that they have no conflict of interest.

\section{Ethical approval}

All procedures performed in this study were in accordance with the ethical standards of the institutional and/or national research committee and with the 1964 Helsinki declaration and its later amendments or comparable ethical standards.

\section{Informed consent}

For this type of study formal consent is not required

\section{Funding}

This study was not funded by any grant.

\section{References}

1. La Vecchia C, Malvezzi M, Bosetti C, Garavello W, Bertuccio P, Levi F, Negri E: Thyroid cancer mortality and incidence: a global overview. Int J Cancer 136:2187-2195 (2015) doi: 10.1002/ijc. 29251 
2. Spinelli C, Strambi S, Bakkar S, Nosiglia A, Elia G, Bertocchini A, Calani C, Leoni M, Morganti R, Materazzi G: Surgical management of diffuse sclerosing variant of papillary thyroid carcinoma. Experience in 25 patients. World J Surg. 44:155-162 (2020) doi: 10.1007/s00268-019-05230-5.

3. Nabhan F, Ringel MD: Thyroid nodules and cancer management guidelines: comparisons and controversies. Endocr Relat Cancer. 24:R13-R26 (2017) doi: 10.1530/ERC-16-0432.

4. Haugen BR, Alexander EK, Bible KC, et al: 2015 American Thyroid Association management guidelines for adult patients with thyroid nodules and differentiated thyroid cancer. Thyroid. 26:1133 (2016) doi: 10.1089/thy. 2015.00207.

5. Tuttle RM, Haugen B, Perrier ND: Updated American Joint Committee on Cancer/Tumor-NodeMetastasis Staging System for Differentiated and Anaplastic Thyroid Cancer (Eighth Edition): What Changed and Why? Thyroid 27:751-756 (2017) doi: 10.1089/thy.2017.0102.

6. Miccoli P, Bakkar S: Surgical management of papillary thyroid carcinoma: an overview. Updates Surg. 69:145-150 (2017) doi: 10.1007/s13304-017-0449-5.

7. Bakkar S, Papavramidis TS, Aljarrah Q, Materazzi G, Miccoli P: Energy-based devices in thyroid surgery-an overview. Gland Surg. 9 (Suppl 1): S14-S17 (2020) doi: 10.21037/ gs.2019.08.05

8. Bakkar S, Macerola E, Aljarrah Q, Proietti A, Materazzi G, Basolo F, Miccoli P: BRAF ${ }^{\mathrm{V} 600 \mathrm{E}}$ mutation: a potential predictor of more than a Sistrunk's procedure in patients with thyroglossal duct cyst carcinoma and a normal thyroid gland. Updates Surg. 71: 701-704 (2019) doi: 10.1007/s13304-01900684-7.

9. Bakkar S, Al-Omar K, AL-Jarrah Q, Al-Dabbas M, Al-Dabbas N, Samara S, Miccoli P: Impact of COVID19 on thyroid cancer surgery and adjunct therapy. Updates Surg 72:867-869 (2020) https://doi.org/10.1007/s13304-020-00833-3.

10. Bilimoria KY, Bentrem DJ, Ko CY, Stewart AK, Winchester DP, Talamonti MS, Sturgeon C: Extent of surgery affects survival for papillary thyroid cancer. Ann Surg 246:375-381 10 (2007).

11. American Thyroid Association (ATA) Guidelines Taskforce on Thyroid Nodules and Differentiated Thyroid Cancer, Cooper DS, Doherty GM, Haugen BR, Kloos RT, Lee SL, Mandel SJ, Mazzaferri EL, Mclver B, Pacini F, Schlumberger M, Sherman SI, Steward DL, Tuttle RM: Revised American Thyroid Association management guidelines for patients with thyroid nodules and differentiated thyroid cancer. Thyroid 19:1167-1214 11 (2009).

12. Adam MA, Pura J, Gu L, Dinan MA, Tyler DS, Reed SD, Scheri R, Roman SA, Sosa JA: Extent of surgery for papillary thyroid cancer is not associated with survival: an analysis of 61,775 patients. Ann Surg 260:601-615 (2014).

13. Adam MA, Pura J, Goffredo P, Dinan MA, Hyslop T, Reed SD, Scheri RP, Roman SA, Sosa JA: Impact of extent of surgery on survival for papillary thyroid cancer patients younger than 45 years. J Clin Endocrinol Metab 100:115-121 (2015) doi:10. 1210/jc.2014-3039.

14. Edge S, Byrd DR, Compton CC, Fritz AG, Greene FL, Trotti A (eds) AJCC Cancer Staging Manual, 7th edn. Springer, New York (2010). 
15. Choi JB, Lee SG, Kim MJ, Kim TH, Ban EJ, Lee CR, Lee J, Kang SW, Jeong JJ, Nam KH, Chung WY, Park CS: Oncologic outcomes in patients with 1-cm to 4-cm differentiated thyroid carcinoma according to extent of thyroidectomy. Head Neck. 41:56-63 (2019) doi: 10.1002/hed.25356.

16. Sawant R, Hulse K, Sohrabi S, Yeo JCL, Pal K, Gibb FW, Adamson R, Nixon IJ: The impact of completion thyroidectomy. Eur J Surg Oncol.45:1171-1174 (2019). doi: 10.1016/j.ejso.2019.03.018. 